\begin{tabular}{|c|l|}
\hline Title & Molecular structure of self-healing poly ampholyte hydrogels analyzed from tensile behaviors \\
\hline Author(s) & Sun, T ao Lin; Luo, Feng; Kurokawa, Takayuki; Karobi, Sadia Nazneen; Nakajima, Tasuku; Gong, Jian Ping \\
\hline Citation & $\begin{array}{l}\text { Soft matter, 11(48), 9355-9366 } \\
\text { https://di.org/10.1039/c5sm01423a }\end{array}$ \\
\hline Issue Date & $2015-12-28$ \\
\hline Doc URL & http://hdl.handle.net/2115/63927 \\
\hline Type & article(author version) \\
\hline File Information & Manuscript-Sun-soft matter.pdf \\
\hline
\end{tabular}

Instructions for use 


\title{
Molecular Structure of Self-healing Polyampholyte Hydrogels Analyzed from Tensile Behaviors
}

\author{
Tao Lin Sun ${ }^{1}$, Feng Luo ${ }^{1}$, Takayuki Kurokawa ${ }^{1}$, Sadia Nazneen Karobi ${ }^{2}$, \\ Tasuku Nakajima ${ }^{1}$, Jian Ping Gong ${ }^{1 *}$ \\ ${ }^{1}$ Laboratory of Soft and Wet Matter, Faculty of Advanced Life Science, Hokkaido \\ University, Sapporo 060-0810, Japan \\ ${ }^{2}$ Laboratory of Soft and Wet Matter, Graduate School of Life Science, Hokkaido \\ University, Sapporo 060-0810, Japan \\ *E-mail: gong@mail.sci.hokudai.ac.jp
}

\begin{abstract}
Recently, charge balanced polyampholytes (PA) have been found to form tough and self-healing hydrogels. This class of physical hydrogels have a very high equilibrated polymer concentration in water (c.a. 40-50 wt\%), and are strongly viscoelastic. They are synthesized by random copolymerization of equal amount of oppositely charged monomers at high concentration, followed by a dialysis process of the small counter-ions and co-ions in water. The randomly distributed, opposite charges of the polymer form multiple ionic bonds of intra- and inter-chains with strength distribution.
\end{abstract} The strong inter-chains bonds, stabilized by topological entanglement, serve as quasi-permanent crosslinking, imparting the elasticity, while the weak bonds, both inter- 
and intra-chains, reversibly break and re-form, dissipate energy to toughen the materials. In this work, we intend to clarify the structure of the physical PA hydrogels from the tensile behaviors of the PA hydrogels. To clarify the structure and its formation mechanism, we analysed the tensile behaviors of the samples before and after the dialysis. We separated the quasi-permanent crosslinking of strong inter-chain bonds and dynamic crosslinking of weak inter-chain bonds by using a combined model that consists of the Upper Convected Maxwell model and the Gent strain hardening model. The model fitting of the tensile behaviors extracts quantitative structure parameters, including the densities of weak and strong inter-chain bonds and the theoretical finite extensibility of polymer chains. Based on the fitting results of the combined model, the structure parameters of partial chain at a fixed observation time, including the Kuhn number, Kuhn length, and chain conformation, are determined using the scaling theory. The effects of monomer concentration at preparation, the effect of dialysis and initial strain rate on the dynamic structure of PA gels, are discussed based on these analyses.

Key words:

Tough hydrogel, Polyampholyte, Ion complex, Sacrificial bonds, Self-healing 


\section{INTRODUCTION}

Hydrogels are crosslinked polymer networks containing abundant of water. Hydrogels had been a class of mechanically weak materials and were paid less attention. Inventions of various kinds of strong and tough hydrogels have largely changed these traditional views and thus substantially broadened the potential applications of hydrogels in various kinds of fields ${ }^{1-3}$. The first work that presented a strategy to develop strong and tough hydrogels is the double-network hydrogel (DN) hydrogels. DN gels consist of two interpenetrating networks, one is stiff and brittle, and the other is soft and stretchable. The stiff and brittle network breaks into fragments by deformation, which effectively dissipates energy to toughen the materials, while the soft and stretchable network maintains the integrity of the materials ${ }^{2}$. Thus, the covalent bonds of the brittle network serve as sacrificial bonds. Based on this double network concept, a serials of tough hydrogels and elastomers have developed ${ }^{4-6}$. Due to breaking of the covalent bonds, DN hydrogels show permanent softening after large deformation. To address this problem, many efforts have been made to develop hydrogels with reversible sacrificial bonds using non-covalent bonds, such as ionic bonds, hydrogen bonds and hydrophobic interaction. Along this line, various hydrogels with high toughness and self-healing properties have been developed ${ }^{7-15}$. These self-healing hydrogels exhibit strong time-dependent behaviors as the result of dynamic breaking and reforming of the 
physical bonds. A theoretical model has recently been developed to describe the kinetics of bond breaking and reforming and its effect on the macroscopic time dependent mechanical behavior of hydrogels ${ }^{16,17}$.

The result of the double network hydrogels suggests a more general strategy to develop tough soft materials, i.e., inducing crosslinking bonds with different level of strength to toughen the materials. Recently, we developed a class of tough and self-healing hydrogels from charge-balanced linear polyampholytes (PAs) that carry opposite charges on the polymer chains ${ }^{10,11,13}$. The PA gels, having $50-60 \mathrm{wt} \%$ of water, are strongly viscoelastic and show excellent mechanical performance, such as high toughness (fracture energy $\sim 4000 \mathrm{~J} / \mathrm{m}^{2}$ ), self-healing, and high fatigue resistance.

These PA hydrogels are synthesized from radical copolymerization of equal amount of oppositely charged ionic monomers at very high concentration to form polymer chains in topological entanglement. As the ability of ionic bond formation depends on the ionic strength of the medium, the as-prepared PA samples, containing large amount of counter ions, are soft, elastic, and highly stretchable gels. By immersing these samples in water, the mobile counter-ions are dialyzed away from the samples, and the formation of interand intra-chains ionic bonds is substantially enhanced. As a result, the gels become substantially rigid and tough after being equilibrated in water. The randomly distributed, 
opposite charges of the polymer form many multiple-ionic-bonds of intra- and inter-chains with strength distribution that is characterized by the relaxation time. At a given observation time, a multiple-ionic-bonds that does not rupture is referred as a strong bond, while a multiple-ionic-bonds that rupturues is referred as a weak bond. So at a given observation time, the strong bonds of inter-chains, stabilized by the topological inter-chain entanglement, serve as permanent crosslinking to maintain the shape of the gel; while the weak bonds, both inter- and intra-chains, reversibly break and re-form to dissipate energy and make the materials tough and self-healing, functioning as reversible sacrificial bonds. Since the structure of the PA gels, that is, the density of topological entanglements and the density of the inter- and intra-chain ionic bonds, strongly depend on the concentration of the polymer at preparation, the mechanical properties of the gels are strongly dependent on the monomer concentration $C_{m}$ at preparation. ${ }^{10,11,13}$

In this work, we intend to quantitatively clarify the dynamic structure of the physical PA hydrogels from the tensile behaviors of the PA hydrogels. For this purpose, we analyzed the tensile behaviors of a serials of PA hydrogels P(NaSS-co-MPTC) synthesized by random copolymerization of equal amount of oppositely charged ionic monomers, anionic monomer p-styrenesulfonate (NaSS) and cationic monomer 
3-(methacryloylamino) propyl-trimethylammonium chloride (MPTC) (Figure 1a). The samples were synthesized at various monomer concentrations, and tensile behaviors of these samples were measured for the as-prepared samples and water-equilibrium samples at varied initial strain rate. We used a viscoelastic model to analyze the tensile data of hydrogels. From the model, the elastic components of strong and weak inter-chain crosslinking at a given observation time can be separated. Based on the structure parameters obtained by the model fitting, we discuss the intra-chain bonds from the finite extensibility of the samples, using the scaling theory. From the structure information, we discuss the effect of dialysis, monomer concentration at preparation, and the observation time on the dynamic molecular structure of the PA hydrogels. This work established a method to reveal the molecular structure of the self-healing, viscoelastic gels from the tensile behaviors. The approach developed here might be applicable to other viscoelastic hydrogel systems.

\section{EXPERIMENTAL SECTION}

\section{PA hydrogels}

The P(NaSS-co-MPTC) PA hydrogels were synthesized using the one-step random copolymerization of an anionic monomer $p$-styrenesulfonate (NaSS) and a cationic 
monomer 3-(methacryloylamino)propyl-trimethylammonium chloride (MPTC) as described in the literature ${ }^{10}$. The gels was synthesized from a mixed aqueous solution with the fixed monomer ratio NaSS:MPTC $=0.52: 0.48$ but different total monomer concentration $\left(C_{m}: 1.3-2.2 \mathrm{M}\right), 0.25 \mathrm{~mol} \% \mathrm{UV}$ initiator, 2-oxoglutaric acid (in relative to $C_{m}$ ), and $0.5 \mathrm{M} \mathrm{NaCl}$. The mixture solution was poured into in a reaction cell consisting of a pair of glass plates with a 3-mm spacing and irradiated with $365 \mathrm{~nm} \mathrm{UV}$ light for 11 hours. After polymerization, the as-prepared gel was immersed in a large amount of water for 1 week to reach equilibrate and to wash away the residual chemicals.

Swelling ratio measurements. The as-prepared PA gels were formed in glass plates with rectangle shapes and then were immersed in water to reach the equilibrium state. The volume ratio $Q_{v}$ was defined as the ratio of the sample volume at water-equilibrium $V$ to that in the as-prepared state $V_{0}, Q_{v}=V / V_{0}$. The water content $c(\mathrm{wt} \%)$ of the equilibrium sample was measured by the weight change upon drying using a freeze-drying process. So the volume fraction of polymer $\phi_{p}$ can be calculated from the equation ${ }^{18}$ : $\phi_{p}^{-1}=1+\left(\frac{c}{1-c}\right) \frac{\rho_{p}}{\rho_{w}}$ where the $\rho_{p}\left(\approx 1.19 \pm 0.02 \mathrm{~g} / \mathrm{cm}^{3}\right)$ and $\rho_{w}\left(\approx 0.998 \mathrm{~g} / \mathrm{cm}^{3}\right)$ are the density of PA and water, respectively. To achieve adequate precision, three measurements were carried out 
on samples of different volumes taken from the same batch.

Tensile test. The tensile stress-elongation measurements were performed on a tensile-compressive tester (Tensilon RTC-1310A, Orientec Co.) in air. The test was carried out on dumbbell-shaped samples of 2-3 $\mathrm{mm}(w)$ thickness with the standard JIS-K6251-7 size $\left(12 \mathrm{~mm}\left(L_{0}\right) \times 2 \mathrm{~mm}(d)\right)$. To prevent water from evaporating from samples, the as-prepared samples were coated with silicone oil during the measurement while equilibrated samples were tested in a moisture environment. The stretching was performed at a constant velocity $v$. The nominal stress $\sigma$ was obtained from the tensile force divided by the initial cross-section area of the sample and the elongation ratio $\lambda$ was obtained from the ratio of stretching length $L=L_{0}+v t$ and the initial length $L_{0}$, where $t$ is the stretching time. The initial strain rate $\dot{\varepsilon}$ was obtained by $v / L_{0}$.

\section{RESULTS}

\section{Effect of dialysis and monomer concentration}

Formation of ion complex structure promotes the shrinkage of hydrogel synthesized at high $C_{m}\left(C_{m}>1.6 \mathrm{M}, Q_{v}=\mathrm{V} / \mathrm{V}_{0}<1\right)$ while gels swell $\left(Q_{v}>1\right)$ at low $C_{m}(<1.6 \mathrm{M})$, as shown in Figure 1b. Here $V$ and $V_{0}$ are the volumes of the gel in their equilibrated state and as-prepared state, respectively. In corresponding to this volume change, the polymer 
volume fraction of the equilibrated gels, $\phi_{p}$, is higher than their as-prepared state when $C_{m}>1.6 \mathrm{M}$, and vice versa (Figure 1c). The deswelling or swelling equilibrium is determined by the balance between the osmotic parts of solvent excluded volume interactions to swell the network, ionic bond formation to contract the network, and elastic energy of polymer chains that is dominated by the density of entanglements and inter-chain bonds. The entanglements and the inter-chain ionic bonds are strongly dependent of the concentration of the polymer at preparation. At low $C_{m}$, the inter-chain ionic bonds formation is suppressed and the solvent excluded volume interactions overcome the electrostatic attraction, resulting in the swelling of gel. While, at high $C_{m}$ the inter-chain ionic bridging overcomes the osmotic swelling and therefore the gels deswell in water. The deswelling behaviour of PA gels due to strong inter-chain bonding is very different from conventional hydrogels that usually swell further from the as-prepared state.

The tensile behaviours of as-prepared hydrogels in the concentrated region $\left(C_{m}=\right.$ $1.3 \sim 2.2 \mathrm{M})$ are depicted in Figure $2 \mathrm{a}$, where $\sigma$ is the nominal stress and $\lambda$ the elongation ratio. The as-prepared gels exhibit the typical features of rubber-like materials and no yielding point is observed during the elongation. High $C_{m}$ results in an increase of modulus and fracture strength, but a decrease in fracture elongation of the 
gels. These behaviors should be attributed to the increase of topological entanglements as well as inter-chain bonds at high $C_{m}$.

After reaching the thermodynamic equilibrium in water, tremendous differences in tensile behaviour between swelling and shrunken hydrogels are observed, as described in Figure $2 \mathrm{~b}$. The hydrogel synthesized at low monomer concentrations $\left(C_{m}: 1.3 \mathrm{M}\right)$ substantially swells in water, and becomes very soft and weak. The samples prepared at high concentration slightly swell $\left(C_{m}: 1.5 \mathrm{M}\right)$ or shrink $\left(C_{m}: 1.7 \sim 2.2 \mathrm{M}\right)$ in water, and they all show enhanced modulus and fracture stress but decreased fracture strain in comparison with their as-prepared state. For shrunk hydrogels $\left(C_{m}: 1.7 \sim 2.2 \mathrm{M}\right)$, clear yielding points are observed. The yielding reveals the internal structure breaking beyond a certain elongation, which dissipates energy and enhances the toughness of hydrogels. Actually, these samples showed large hysteresis during the cyclic test. ${ }^{10}$

The Young's modulus $E$, fracture stress $\sigma_{b}$, and fracture elongation $\lambda_{b}$ of as-prepared and water-equilibrated PA hydrogels are summarized in Figure 3. Increase in $C_{m}$ dramatically enhances $E$ and $\sigma_{b}$ for as-prepared gels, while $E$ becomes constant for shrunk samples $\left(C_{m}>1.6 \mathrm{M}\right)$ but $\sigma_{b}$ increases with $C_{m}$ in the whole range for equilibrium gels in water (Figures 3a and 3b). Compared with as-prepared hydrogels that have low moduli $(E=0.0047-0.070 \mathrm{MPa})$, the hydrogels after deswelling become 
very rigid $(E=1.5-2.0 \mathrm{MPa})$, indicating the formation of substantial amount of inter-chain ionic bonds in water, as the density of topological entanglement does not change with the dialysis. These equilibrated hydrogels are strong, as shown by their high tensile fracture stress $\sigma_{b}=0.88-1.93 \mathrm{MPa}$, which is several times larger than that of the corresponding as-prepared hydrogels $\left(\sigma_{b}=0.033-0.46 \mathrm{MPa}\right)$.

The equilibrated hydrogels have smaller $\lambda_{b}$ than that of as-prepared hydrogels due to the formation of strong inter-chain ionic bonds, which limits the finite extensibility. The interesting phenomenon is that not only the fracture stress $\sigma_{b}$ but also the fracture elongation $\lambda_{b}$ of these equilibrated samples increases with the increase of $C_{m}$, in contrast to the behaviour of the as-prepared gels that show decrease of $\lambda_{b}$ with $C_{m}$ (Figure $3 \mathrm{c}$ ). This result is attributed to the enhanced toughness of the equilibrated samples with $C_{m}$, which effectively suppresses the crack propagation and thus increases the $\lambda_{b}{ }^{10,11,13}$

The features of rubber elasticity can be further analysed by the phenomenological Mooney-Rivlin equation, ${ }^{19-21}$

$$
\sigma_{\text {red }}=\frac{\sigma}{\lambda-\lambda^{-2}}=2 C_{1}+2 C_{2} \frac{1}{\lambda}
$$

where $\sigma_{\text {red }}$ is the reduced stress, $C_{1}$ and $C_{2}$ are materials constants. The $2 C_{1}$ is equal to the shear modulus $(=E / 3)$, and $C_{2}$ is related to the strain softening $\left(C_{2}>0\right)$ or hardening $\left(C_{2}<0\right)$ beyond the Gaussian elasticity region. To catch the features of the gels at large 
deformation, we plot $\sigma_{\text {red }}$ against $\lambda^{-1}$ for samples with different $C_{m}$, which are shown in Figure $2 \mathrm{c}, 2 \mathrm{~d}$ for the as-prepared samples and equilibrated samples, respectively. In the case of the as-prepared gels, at $\lambda^{-1}>0.2, \sigma_{\text {red }}$ is almost independent of $\lambda^{-1}$ for low $C_{m}$ samples $\left(C_{m}=1.3-1.7 \mathrm{M}\right)$ or only slightly increases with the increase of $\lambda^{-1}$ for high $C_{m}$ samples $\left(C_{m}=1.9,2.2 \mathrm{M}\right)$. This result indicates that the low $C_{m}$ samples are purely rubber elastic $\left(C_{2}=0\right),{ }^{20}$ while high $C_{m}$ samples have slight strain softening (for example, $C_{2}=0.0056$ for $C_{m}=2.2 \mathrm{M}$ ). The latter should be attributed to the breakage of inter-chain ionic bonds. ${ }^{20}$ At the large deformation $\left(\lambda^{-1}<0.2\right)$, distinct strain hardening due to finite extensibility of polymer chains is observed, which is the evidence of the presence of quasi-permanent crosslinking. Given the fact that a simple entangled polymer solution with no polymer association does not form gel at the observation condition, the results of Figure $2 \mathrm{a}, 2 \mathrm{c}$ indicate that even before the dialysis of the samples, some strong inter-chain ionic bonds already exist to form gels. The relationship of $E \sim C_{m}^{4.9}$ observed in Figure 3a suggests that these strong inter-chain ionic bonds are formed at the topological entanglement points.

For the water-equilibrated samples, the Mooney-Rivlin plots show substantial strain softening for all the samples, except for that prepared at low monomer concentration $\left(C_{m}: 1.3 \mathrm{M}\right)$ that shows large swelling (Figure $\left.2 \mathrm{~d}\right)$. The strain softening 
occurs more prominently at high $C_{m}$. The results indicate that more large amount of inter-chain bonding is broken during tensile deformation for samples prepared at high $C_{m}$. This breakage of weak ionic bonds yields energy dissipation to toughen the gels. The strong strain-softening behaviour of the equilibrated gels is in consistent with the large hysteresis observed in the load-unloading cyclic test. ${ }^{10}$

\section{Effect of strain rate}

As discussed earlier, the strength of a dynamic ionic bond strongly depends on the observed time or strain rate, and the mechanical performance of PA hydrogels depends on the strain rate. We analysed the tensile behaviours of a PA hydrogel $\left(C_{m}: 2.1 \mathrm{M}\right)$ with varied initial strain rate $\dot{\varepsilon}\left(0.0069 \sim 0.69 \mathrm{~s}^{-1}\right)$, and the typical results for the as-prepared sample and the water equilibrated sample are shown in Figures $4 a$ and $4 b$, respectively. The variations of Young's modulus $E$, fracture stress $\sigma_{b}$, and fracture elongation $\lambda_{b}$ with initial strain rate $\dot{\varepsilon}$, are summarized in Figure 5. The as-prepared gel shows relatively weak strain rate dependence of $E, \sigma_{b}$, and $\lambda_{b}$. This again indicates that the as-prepared sample has a rubbery feature with very weak viscoelasticity. On the other hand, the $E$ and $\sigma_{b}$ of the equilibrated sample increase with the increase of $\dot{\varepsilon}$, following the nice power law relations, $E \sim \dot{\varepsilon}^{0.38}$ and $\sigma_{b} \sim \dot{\varepsilon}^{0.17}$. The behaviour of $E$ is in agreement with 
our previous report. ${ }^{11}$ At a high initial strain rate, more inter-chain bonds serve as strong bonds to increase the strength of the gels. The $\lambda_{b}$ decreases with the increase of initial strain rate $\dot{\varepsilon}$, which is consistent with the increase of strong bonds at high initial strain rate. These strong initial strain rate dependences indicate the viscoelastic feature of the equilibrated sample. It should be noted that the true strain rate $v / L$ decreases with increase of stretching, and the true strain rate at breakage of samples, given by $v /\left(L_{0} \lambda_{b}\right)$, was about $1 / 10$ of the initial strain rate $v / L_{0}$ since $\lambda_{b}$ was $\sim 10$.

As shown by the Mooney plot curves in Figures 4c and 4d, the as-prepared gel shows weak strain softening at low deformation, as like common elastic hydrogels. This indicates that there are few weak inter-chain bonds that break during deformation in this gel. In contrast, the equilibrated sample shows strong strain softening, which becomes more prominent at high initial strain rate. This result indicates that there are abundant of weak inter-chain bonds that break during deformation. The increase of strain softening with the initial strain rate indicates that more weak bonds are ruptured by deformation.

\section{DISCUSSIONS}

\section{Viscoelastic model}

At a given observation time, the strong inter-chain bonds, stabilized by topological 
entanglements, serve as the permanent crosslinkers, while the weak inter-chain bonds serve as temporary crosslinkers. The weak ionic bonds, both inter- and intra-chains, break and reform reversibly, dissipating energy and contributing to the toughness of hydrogels. So we need to separate the strong bonds and weak bonds, both of inter-chain and intra-chains. Many theoretical models have been proposed for the viscoelastic behaviors of materials. 16, 22, 23 Among several models tested, we found that the viscoelastic model proposed by the Creton et al. can well fit this system. ${ }^{23}$ This model assumes that the system consists of a parallel combination of the Upper Convected Maxwell (UCM) model for the description of viscoelastic flow and Gent strain hardening model for the entropic elasticity of finite extensibility of polymer chains (Figure 6). The sum of the two contributions to the nominal stress $\sigma_{N}$ from the UCM element and the Gent element can be described as

$$
\sigma_{N}(\lambda)=\sigma_{N, v}(\lambda)+\sigma_{N, e}(\lambda)
$$

where

$$
\sigma_{N, v}(\lambda)=\left(\frac { 2 G _ { v } D _ { e } } { 1 - 2 D _ { e } } \left(1-\exp \left(-\frac{\left(1-2 D_{e}\right)}{D_{e}}(\lambda-1)\right)+\frac{G_{v} D_{e}}{1+D_{e}}\left(1-\exp \left(-\frac{\left(1+D_{e}\right)}{D_{e}}(\lambda-1)\right)\right) \lambda^{-1}\right.\right.
$$

for the viscoelastic stress, and

$$
\sigma_{N, e}(\lambda)=\left(\frac{G_{e}}{1-\frac{\lambda^{2}+2 \lambda^{-1}-3}{J_{m}}}\left(\lambda^{2}-\lambda^{-1}\right)\right) \lambda^{-1}
$$

for the entropic elastic stress. Here, $\lambda$ is the elongation ratio, $G_{v}$ is the initial shear 
modulus of the viscoelastic part, stemming from the contribution of weak inter-chain bonds that rupture during deformation. $D_{e}$ is the Deborah number (the product of the relaxation time of the viscous component and the strain rate). $G_{e}$ is the shear modulus at small strain from the elastic part, stemming from the contribution of strong inter-chain bonds as well as the topological inter-chain entanglement that do not flow during the deformation, serving as quasi-permanent crosslinker. $J_{m}$ is the maximum allowable value of the first strain invariant representing the theoretical finite extensibility of the network chains, which can be written as

$J_{m}=\lambda_{m}^{2}+2 \lambda_{m}^{-1}-3$

where $\lambda_{m}$ represent the theoretical finite extensibility of polymer chains. In this system, $\lambda_{m}$ depends on both the weak inter- and intra-chain ionic bonds that rupture during deformation. Eq. 2 is determined by four parameters $G_{e}, \lambda_{m}, G_{v}$, and $D_{e}$. Since the Young's modulus $E=3\left(G_{e}+G_{v}\right)$, we can fit the tensile stress-strain data with the model using 3 independent parameters and the Young's modulus $E$ estimated from the tensile behaviour. From the elasticity theory, we can relate the modulus $G$ to the chain density $v$ by $G=\phi_{p} v k_{B} T$

Here, $v$ is the chain density without solvent $\left(\phi_{p}=1\right), k_{B}$ the Boltzmann constant, and $T$ 
the temperature. So, we can discuss the density of inter-chain ionic bonds from $G_{e}$ (quasi-permanent strong bonds) and $G_{v}$ (viscoelastic weak bonds) for a specific observation time. From $\lambda_{m}$ we can discuss the intra-chain ionic bonds.

A represented stress-elongation curve of hydrogels and the simulated curves of the two individual contributions are shown in Figure 6. The good fit of the simulated curves to the experimental curve indicates that the tensile behaviours of the PA hydrogels could be well expressed by such a combined model. We performed a systematic fitting to the gels prepared at various monomer concentrations $C_{m}$ and measured at a given initial strain rate $\left(0.14 \mathrm{~s}^{-1}\right.$, Figures $\left.2 \mathrm{a}, \mathrm{b}\right)$ and the results are shown in Figure 7.

At a given initial strain rate $\left(0.14 \mathrm{~s}^{-1}\right)$, the $G_{v}$ and $G_{e}$ increase abruptly above a critical $C_{m}>1.4 \mathrm{M}$ by dialysis (Figures $7 \mathrm{a}, \mathrm{b}$ ). This indicates that both the weak and strong inter-chain bonds are formed substantially by the dialysis, especially above the critical concentration $C_{m}$. This result is consistent with the volume change by dialysis (Figure 1b). While increasing in $C_{m}$ enhances $G_{v}$ and $G_{e}$ of the as-prepared gels, the equilibrium gels show almost constant $G_{v}$ and $G_{e}$, independent of $C_{m}$ for high $C_{m}$ samples $\left(C_{m}>1.6\right.$ M). Theoretical finite extensibility $\lambda_{m}$ against $C_{m}$ for the as-prepared gel and equilibrated gel are shown in Figure $7 \mathrm{c}$. Decrease in $\lambda_{m}$ with increasing of $C_{m}$ is obvious in the as-prepared gel while an inverse tendency is observed for the 
equilibrated gel, in agreement with the experimental results of $\lambda_{b}$ in Figure $3 \mathrm{c}$. The results show that although many weak bonds convert into strong bonds after dialysis, the finite extensibility $\lambda_{m}$ decreases only slightly. This is a specific feature of this tough PA hydrogels. Before dialysis, the gel is more elastic and has low resistance against crack propagation, while after dialysis, the gel becomes highly resistant against the crack propagation. This is considered to increase the extensibility $\lambda_{m}$.

The $G_{v} / G_{e}$ indicates the ratio of polymer chains crosslinked by temporary weak bonds and quasi-permanent strong bonds. The value of $G_{v} / G_{e}$ also indicates the average number of weak bonds between two neighbouring strong bonds. For the as-prepared gels, $G_{v} / G_{e}$ varies from 1.5 to 0.5 with increasing of $C_{m}$. After dialysis, $G_{v} / G_{e}$ is about 4 5 for $C_{m}>1.6 \mathrm{M}$, indicating that more inter-chain weak bonds are formed in relative to the strong bonds at the observation strain rate (Figure 7d). The Deborah number $D_{e}$ in the fitting serves as the adjusting parameter. We found that $D_{e}$ is always within the range of subunit (Supporting information, Figure S1a).

The polymer partial chain densities from the elastic part $v_{\mathrm{v}}$ and viscoelastic part $v_{\mathrm{e}}$ for gels prepared at various $C_{m}$ are estimated from Equation 6 and the results are shown in Figure $7 \mathrm{e}$ and $\mathrm{f}$, respectively. The $v_{e}$ in the as-prepared gels increase with the increase of $C_{m}$, roughly following the power law relations, $v_{e} \sim C_{m}^{5.1}$. The large index value of the 
power law of $v_{e}$ is due to the increase of topological entanglement with increase in $C_{m}{ }^{24}$ However, except at the lowest $C_{m}$, the equilibrated gels show high but almost constant values of $v_{e}$ and $v_{v}$, independent of $C_{m}$. This phenomenon can be understood as that at such high concentration, the partial chain becomes too short to form the intra-chain ionic bonds, which is limited by the chain rigidity, and thus the inter-chain complexation is energetically favoured to occur.

As the structure of the PA hydrogels are dynamic, these fitted parameters also strongly depend on the observation time. The fitting results for the gels prepared at a constant monomer concentration $C_{m}$ and measured at different initial strain rate (Figures $4 \mathrm{a}, \mathrm{b}$ ) are shown in Figure 8. For the same $C_{m}(=2.1 \mathrm{M})$, when the initial strain rate varies from 0.0069 to $0.69 \mathrm{~s}^{-1}, G_{v}$ and $G_{e}$ increase with the initial strain rate, following power law relations. Since the as-prepared gel is quite elastic, the initial strain rate dependences are weak $\left(G_{v} \sim \dot{\varepsilon}^{0.10}\right.$ and $\left.G_{e} \sim \dot{\varepsilon}^{0.11}\right)$. The equilibrated gel show more strong initial strain rate dependence $\left(G_{v} \sim \dot{\varepsilon}^{0.41}\right.$ and $\left.G_{e} \sim \dot{\varepsilon}^{0.23}\right)$, featuring its viscoelastic nature (Figure 8a, b). The increase of $G_{v}$ with the initial strain rate indicates that fully relaxed ionic bonds at a low initial strain rate convert into weak bonds at a high initial strain rate, while the increase of $G_{e}$ indicates that weak bonds at relatively low initial strain rate converts into strong bonds at high initial strain rate. The ratio of $G_{v} / G_{e}$, which stands for the ratio of 
crosslinking density by weak bonds and quasi-permanent crosslinkers, is almost independent of the initial strain rate for the as-prepared sample, while it increases with the initial strain rate for the equilibrated sample (Figure 8d).

The theoretical finite extensibility $\lambda_{m}$ of the as-prepared gel is almost constant, independent of the initial strain rate. This is consistent with the elastic feature of the gel. On the other hand, the $\lambda_{m}$ of the equilibrated gel decreases abruptly at the high initial strain rate (Figure 8c). This should be related to the increase of the strong bond density at the high initial strain rate. The values of Deborah number, $D_{e}$, at different initial strain rates are also within the range of subunit (Supporting information, Figure S1b).

\section{Intra-chain ionic bonds}

As discussed previously, the PA also forms intra-chain ionic bonds that do not contribute to $G_{v}$ and $G_{e}$ but strongly influence $\lambda_{m}$. These intra-chain ionic bonds should rupture by stretching. Therefore, the behaviors of intra-chain bonds substantially influence the finite extensibility. In this section, we discuss this effect. Here, we simply assume that the deformation is affine and the theoretical finite extensibility $\lambda_{m}$ is affine extension ratio of a partial chain between the quasi-permanent crosslinkers, that is, the ratio of chain length at effectively finite extensibility to the initial size $R$ of the partial 
chain that is in global state (Figure 9). Without losing the generality, the latter can be expressed as $R=b_{k} N_{k}{ }^{\alpha}$. Here, $b_{k}$ is the Kuhn length, $N_{\mathrm{k}}$ the Kuhn number between quasi-permanent crosslinkers, and the $\alpha$ is the scaling exponent, representing the conformation of PA chains. $\alpha=0.6$ represents a flexible chain of random coil conformation in a good solvent and $\alpha=0.3$ represents globule conformation of a chain in a poor solvent. ${ }^{24}$ Assuming that all the intra-chain bonds are weak bonds and they break at the ultimate extension ratio, then we have,

$\lambda_{m}=\frac{b_{k} N_{k}}{b_{k} N_{k}^{\alpha}}=N_{\mathrm{k}}^{1-\alpha}$

The elastic modulus from the contribution of quasi-permanent crosslinking is

$G_{e}=\phi_{p} v_{e} k_{B} T=\frac{1}{\left(b_{k} N_{k}^{\alpha}\right)^{3}} k_{B} T$

The volume fraction of polymer $\phi_{p}$ is related to the partial chain parameter $N_{k}$ and $b_{k}$ as

$\phi_{p}=\frac{b_{k}{ }^{3} N_{\mathrm{k}}}{\left(b_{k} N_{k}^{\alpha}\right)^{3}}=N_{k}^{1-3 \alpha}$

Using $\lambda_{m}$ and $G_{e}$ determined by the viscoelastic model fitting and $\phi_{p}$ by experiment measurement, we can estimate the three structure parameters, $b_{k}, N_{\mathrm{k}}$, and $\alpha$, from Eqs. (7), (8) and (9). Figure 10 shows these structure parameters for the PA hydrogels prepared at various monomer concentrations $C_{m}$.

Figures 10a shows that, for the as-prepared samples, the scaling exponent $\alpha=0.40$, independent of $C_{m}$ at preparation. This indicates that the polymer chains are in relatively 
collapsed state, close to globule conformation $(\alpha=0.3)$, as a result of intra-chain ionic bond formation. While, for the equilibrated samples after dialysis, $\alpha$ is $C_{m}$-dependent. For the sample of $C_{m}=1.3 \mathrm{M}, \alpha$ is around 0.6 , corresponding to the random coil conformation. The $\alpha$ value decreases with increase of $C_{m}$, approaching 0.4 at high $C_{m}$. This behaviour of $\alpha$ is well related to the volume change after dialysis (Figure 1b). For swollen samples, $\alpha$ is larger than 0.5 while for the deswollen samples $\alpha$ is 0.4 . At low $C_{m}$, the inter-chain ionic bonds formation is suppressed and the solvent excluded volume interactions overcome the electrostatic attraction, resulting in a flexible chain with the random coil conformation. While, at high $C_{m}$ the inter-chain ionic bridging overcomes the osmotic swelling and therefore polymer chains exhibit a globule-like conformation. The $\alpha=0.4$ is slightly larger than the polyampholyte theory that predicts $\alpha=0.3 .^{25,26}$

The as-prepared gels show dramatic decrease of Kuhn number $N_{\mathrm{k}}$ from 450 to 130 with increase of $C_{m}$ (Figures 10b), which can be related to the enhanced entanglements and inter-chain ionic bonds density with the increase of $C_{m}$. In contrast, the equilibrated gels have a constant Kuhn number $N_{\mathrm{k}}(\sim 100)$, independent of $C_{m}$. This is understandable since $v_{e}$ is independent of $C_{m}$, as shown in Figure $7 \mathrm{f}$. The smaller $N_{\mathrm{k}}$ of the equilibrated gels than that of the as-prepared gels can be related to the enhanced inter-chain bonds. 
An interesting observation in Kuhn length $b_{k}$ is that the value in the as-prepared gel is always larger than that in the equilibrated gel, as shown in Figure 10c, which indicates that the polymer chains become much soft after dialysis. According to the theoretical study in stiff PA chains, ${ }^{27}$ the total persistence length of PA chains $l_{p}$ can be written as the sum of the bare persistence length $l_{0}$, arising from the intrinsic stiffness of the chain, and the contribution from charges in the polyampholyte chains $l_{P A}: l_{p}=l_{0}+l_{P A}$. When we consider that the PA is overall neutral, the contribution to the $l_{P A}$ from the charge fluctuations $(\delta \sigma)^{2}$, giving rise to an effective attractive interaction, is proportional to $-(\delta \sigma)^{4} \kappa^{-1}$, where $\kappa^{-1}$ is the Debye screening length. The effective persistence length is reduced since the polyampholyte contribution $l_{P A}$ is intrinsically negative. As a consequence, the local polymer chains of the as-prepared state is much stiffer than that of equilibrated gel prepared at the same $C_{m}$ since the former has a short $\kappa^{-1}$ due to the presence of added salt as well as counter ions. While, the decrease in Kuhn length $b_{k}$ of as-prepared gel with the increase of $C_{m}$ may stem from the results of the competition of Debye length $\kappa^{-1}$ and charge fluctuations $(\delta \sigma)^{2}$.

As the as-prepared gel is quasi-elastic, these structural parameters do not depend on initial strain rate (Figure 11). In contrast, for the equilibrated gel, which is viscoelastic, the declines in $N_{\mathrm{k}}$ and $b_{k}$ at the high initial strain rate are observed while $\alpha$ keeps 
constant (Figures 11a, b and c). At a high initial strain rate, more inter-chain bonds serve as strong bonds to increase their quasi-crosslinker density so that $N_{\mathrm{k}}$ decreases. And this enhanced attractive interaction further contributes to reduce their effective persistence length.

\section{CONCLUSIONS}

The essential features of the structure clarified are schematically summarized in Figure 12. In the as-prepared state, the polyampholyte chains already formed some strong inter-chain ionic bonds to give soft, elastic gels. Considering the presence of large amount of co-ions and counter-ions, these inter-chain ionic bonds are considered to be located around the topological entanglement points. Since the partial chains between the quasi-crosslinking points are found to be in globule conformation, some intra-chain association should also exist in the as-prepared samples. However, these intra-chain bonds should be very weak with bond exchange time much faster than the observation time, as only very weak initial strain rate dependence was observed.

By dialysis, removing of the co-ions and counter-ions from the samples substantially enhances the ionic bond formation, whereupon the polymer concentration governs the competition between the intra-chain and inter-chain complexation. At a high enough 
concentration that the inter-chains distance becomes smaller than the Kuhn length of the chain, the inter-chain complexation is energetically more favourable to occur. As a result, the water equilibrated gels synthesized at high concentration possess both high stiffness and toughness. Since the strong intra-chain bonds reduce the stretching ability and have no contribution to modulus and toughness, elimination of the strong intra-chain bonds is important for the stiff, tough, yet stretchable gels. The understanding of the structure formation process will give insight to design controlled dialysis process for tough gels in future.

\section{ACKOWLEDGEMENT}

This research was funded by ImPACT Program of Council for Science, Technology and Innovation (Cabinet Office, Government of Japan).

\section{REFERENCES}

1. K. Haraguchi and T. Takehisa, Adv Mater, 2002, 14, 1120-1124.

2. J. P. Gong, Y. Katsuyama, T. Kurokawa and Y. Osada, Adv Mater, 2003, 15, 1155-+.

3. T. Huang, H. G. Xu, K. X. Jiao, L. P. Zhu, H. R. Brown and H. L. Wang, Adv Mater, $2007,19,1622^{-+}$.

4. T. Nakajima, H. Sato, Y. Zhao, S. Kawahara, T. Kurokawa, K. Sugahara and J. P. Gong, Adv Funct Mater, 2012, 22, 4426-4432.

5. E. Ducrot, Y. L. Chen, M. Bulters, R. P. Sijbesma and C. Creton, Science, 2014, 344, 
186-189.

6. M. J. Palmeri, K. W. Putz and L. C. Brinson, Acs Nano, 2010, 4, 4256-4264.

7. D. C. Tuncaboylu, M. Sari, W. Oppermann and O. Okay, Macromolecules, 2011, 44, 4997-5005.

8. S. E. Bakarich, G. C. Pidcock, P. Balding, L. Stevens, P. Calvert and M. I. H. Panhuis, Soft Matter, 2012, 8, 9985-9988.

9. J. A. Neal, D. Mozhdehi and Z. B. Guan, JAm Chem Soc, 2015, 137, 4846-4850.

10. T. L. Sun, T. Kurokawa, S. Kuroda, A. Bin Ihsan, T. Akasaki, K. Sato, M. A. Haque, T. Nakajima and J. P. Gong, Nat Mater, 2013, 12, 932-937.

11. F. Luo, T. L. Sun, T. Nakajima, T. Kurokawa, Y. Zhao, A. Bin Ihsan, H. L. Guo, X. F. Li and J. P. Gong, Macromolecules, 2014, 47, 6037-6046.

12. F. Luo, T. L. Sun, T. Nakajima, T. Kurokawa, Y. Zhao, K. Sato, A. Bin Ihsan, X. F. Li, H. L. Guo and J. P. Gong, Adv Mater, 2015, 27, 2722-+.

13. A. Bin Ihsan, T. L. Sun, S. Kuroda, M. A. Haque, T. Kurokawa, T. Nakajima and J. P. Gong, J Mater Chem B, 2013, 1, 4555-4562.

14. J. Y. Sun, X. H. Zhao, W. R. K. Illeperuma, O. Chaudhuri, K. H. Oh, D. J. Mooney, J. J. Vlassak and Z. G. Suo, Nature, 2012, 489, 133-136.

15. K. Mayumi, A. Marcellan, G. Ducouret, C. Creton and T. Narita, Acs Macro Lett, 2013, 2, 1065-1068.

16. R. Long, K. Mayumi, C. Creton, T. Narita and C. Y. Hui, Macromolecules, 2014, 47, 7243-7250.

17. E. B. Stukalin, L. H. Cai, N. A. Kumar, L. Leibler and M. Rubinstein, Macromolecules, 2013, 46, 7525-7541.

18. H. Itagaki, T. Kurokawa, H. Furukawa, T. Nakajima, Y. Katsumoto and J. P. Gong, Macromolecules, 2010, 43, 9495-9500.

19. T. Wang, D. Liu, C. X. Lian, S. D. Zheng, X. X. Liu and Z. Tong, Soft Matter, 2012, 8, 774-783.

20. R. E. Webber, C. Creton, H. R. Brown and J. P. Gong, Macromolecules, 2007, 40, 2919-2927.

21. D. C. Lin, J. F. Douglas and F. Horkay, Soft Matter, 2010, 6, 3548-3561.

22. C. Y. Hui and R. Long, Soft Matter, 2012, 8, 8209-8216.

23. F. Deplace, M. A. Rabjohns, T. Yamaguchi, A. B. Foster, C. Carelli, C. H. Lei, K. Ouzineb, J. L. Keddie, P. A. Lovell and C. Creton, Soft Matter, 2009, 5, 1440-1447.

24. M. Rubinstein and R. H. Colby, Polymer Physics, Oxford University Press, New York, 2003.

25. A. V. Dobrynin, R. H. Colby and M. Rubinstein, J Polym Sci Pol Phys, 2004, 42, 
3513-3538.

26. P. G. Higgs and J. F. Joanny, J Chem Phys, 1991, 94, 1543-1554.

27. B. Y. Ha and D. Thirumalai, J Phys Ii, 1997, 7, 887-902. 


\section{Figure 1}

(a)

NaSS

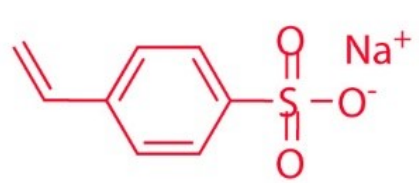

MPTC<smiles>C=C(C)C(=O)NCCC[N+]1(Cl)CC1</smiles>

(b)

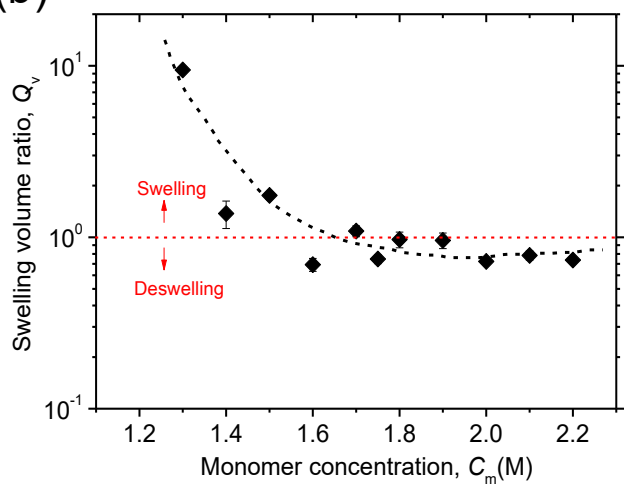

(c)

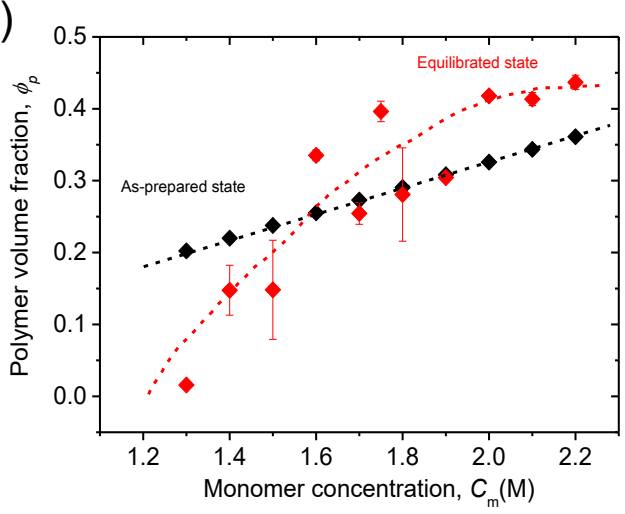

Figure 1. (a) Chemical structures of ionic monomers used in this work. (b) The deswelling of the hydrogels in water depends on the monomer concentration $C_{m}$ in polymerization. The volume ratio of the gels at equilibrated state to that in the as-prepared state $Q_{v}$ is against $C_{m}$. (c) the volume fraction of polymer in the gels $\phi_{p}$ against $C_{\mathrm{m}}$. The data of swelling volume ratio in Figure $1 \mathrm{~b}$ was cited from literature. ${ }^{10}$ 


\section{Figure 2}

(a)

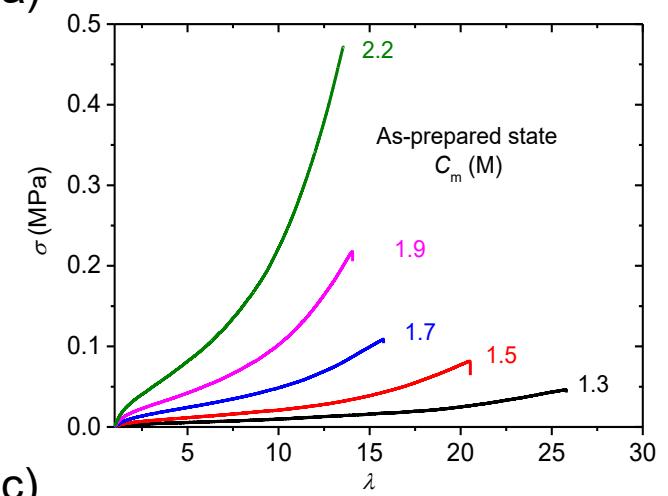

(c)

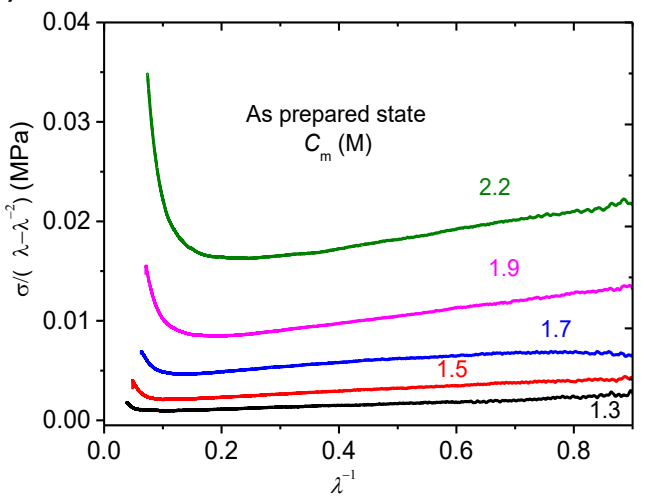

(b)

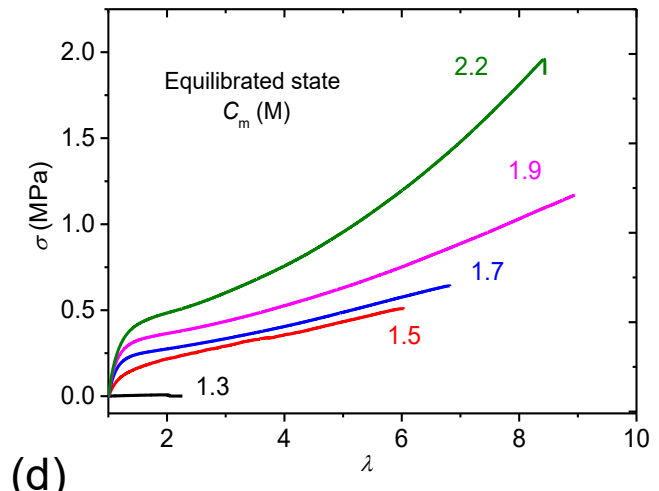

(d)

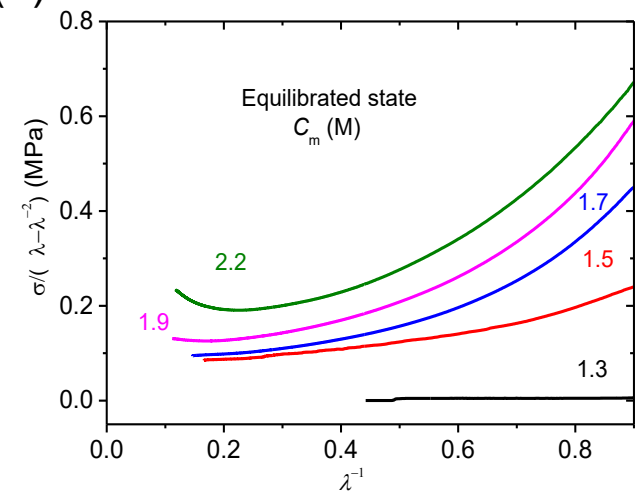

Figure 2. Tensile behaviours (a, b) and corresponding Mooney-Rivlin curves (c, d) of polyampholyte hydrogels synthesized at different monomer concentration $C_{m}$ in their as-prepared state $(a, c)$ and water-equilibrium state $(b, d)$. The measurements were performed at an initial strain rate $0.14 \mathrm{~s}^{-1}$. Figure $2 \mathrm{~b}$ was cited from the literature. ${ }^{10}$ 


\section{Figure 3}

(a)

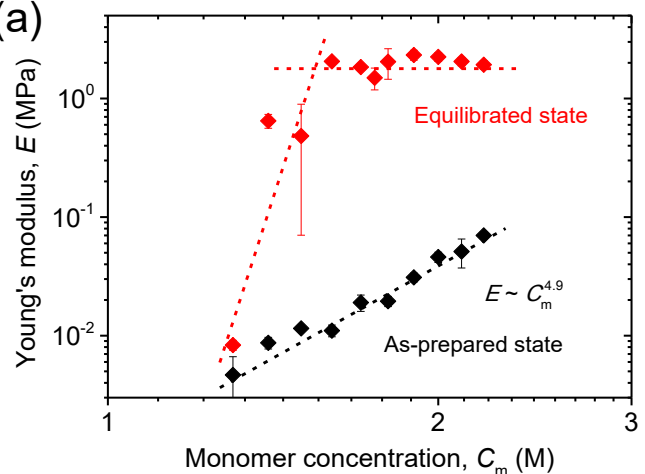

(c)

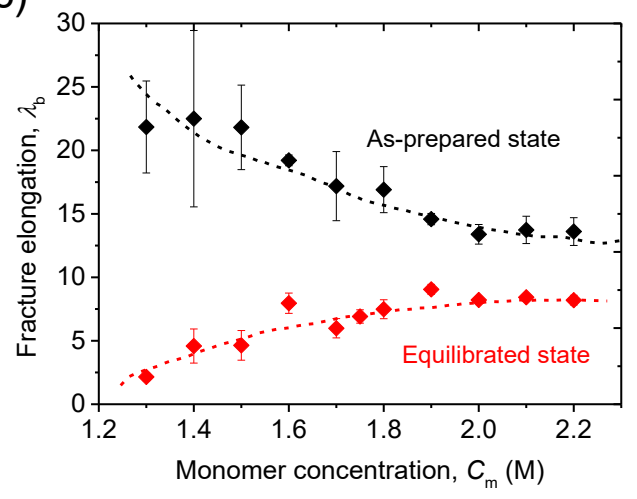

(b)

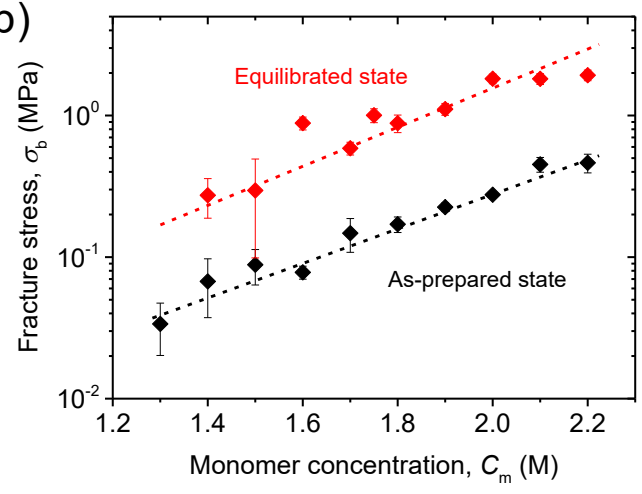

Figure 3. Monomer concentration $C_{\mathrm{m}}$ dependence of Young's modulus $E$ (a), fracture stress $\sigma_{b}(\mathrm{~b})$, and fracture elongation $\lambda_{b}$ (c) of polyampholyte hydrogels in their as-prepared and water-equilibrated state. The measurements were performed at an initial strain rate $0.14 \mathrm{~s}^{-1}$. 


\section{Figure 4}

(a)

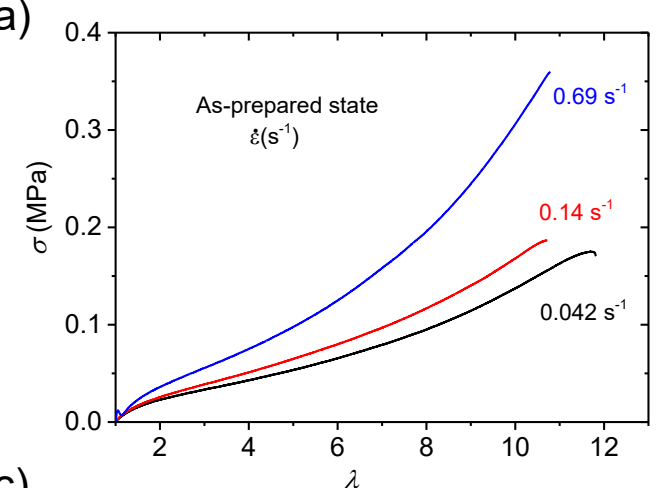

(c)

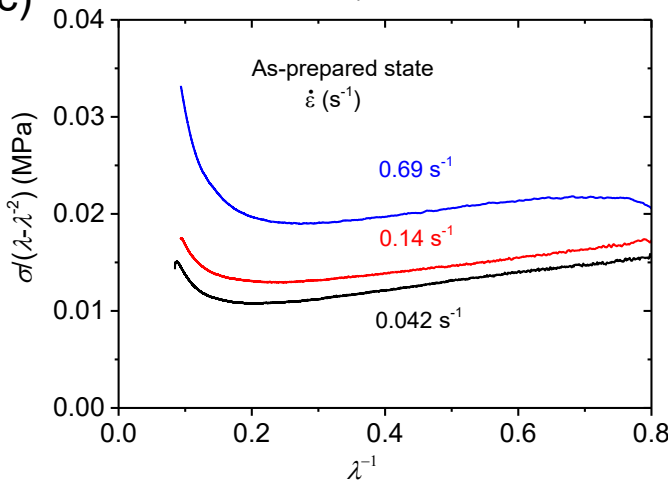

(b)

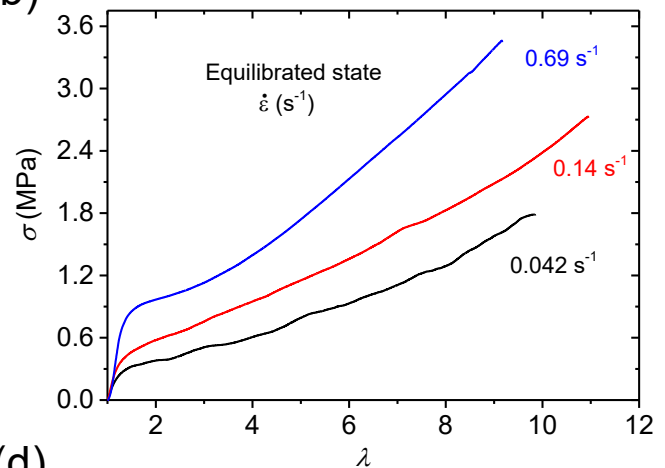

(d)

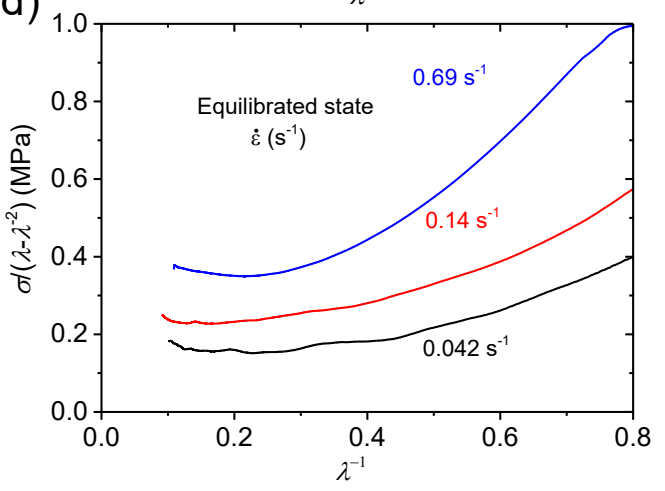

Figure 4. Tensile behaviour (a, b) and corresponding Mooney-Rivlin curves (c, d) of a polyampholyte hydrogel $\left(C_{m}=2.1 \mathrm{M}\right)$ measured at various initial strain rates in its as-prepared state $(a, c)$ and water-equilibrated state $(b, d)$. Figure $4 b$ was cited from the literature. ${ }^{10}$ 


\section{Figure 5}

(a)
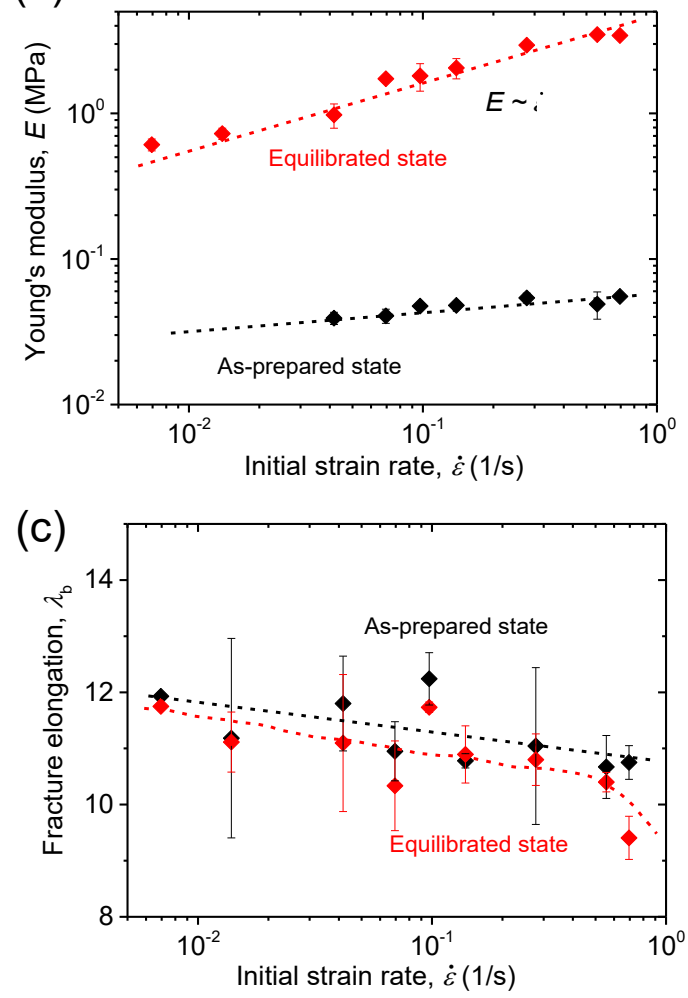

(b)

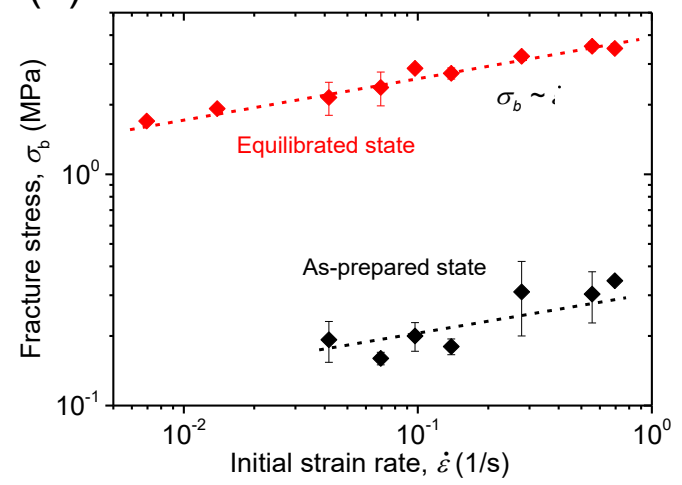

Figure 5. Initial strain rate $\dot{\varepsilon}$ dependence of Young's modulus $E$ (a), fracture stress $\sigma_{b}$ (b), and fracture elongation $\lambda_{b}$ (b) of a polyampholyte hydrogel $\left(C_{m}=2.1 \mathrm{M}\right)$ in its as-prepared and equilibrated state. 


\section{Figure 6}

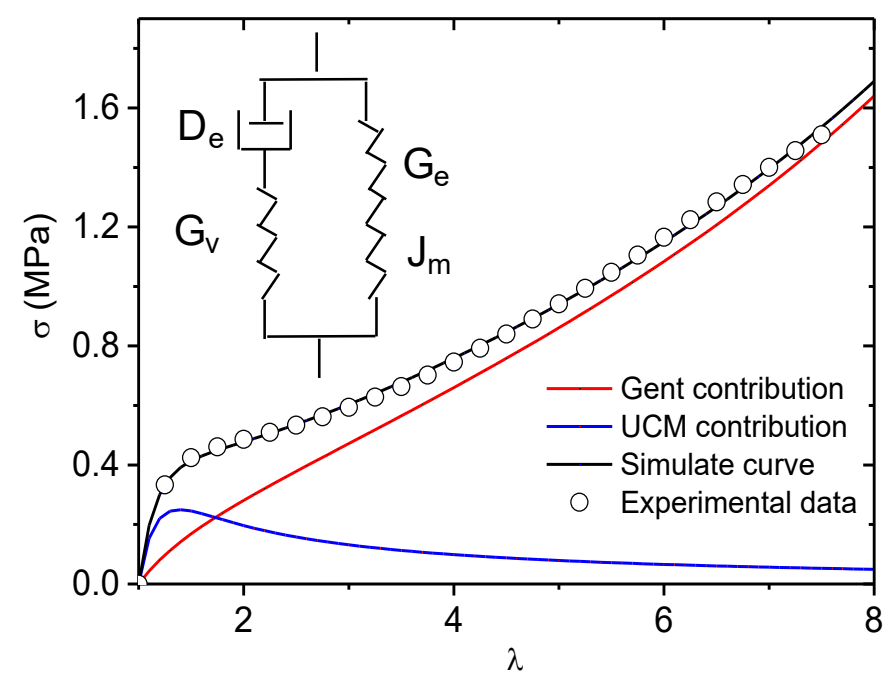

Figure 6. Typical tensile behaviour of equilibrated hydrogel in water (experimental data, $C_{m}=2.0 \mathrm{M}$ ), and simulated sum of contribution (simulate curve), and their individually contributions from the viscous part (UCM) and elastic part (Gent). Inset is the sketch of viscoelastic model. 


\section{Figure 7}

(a)

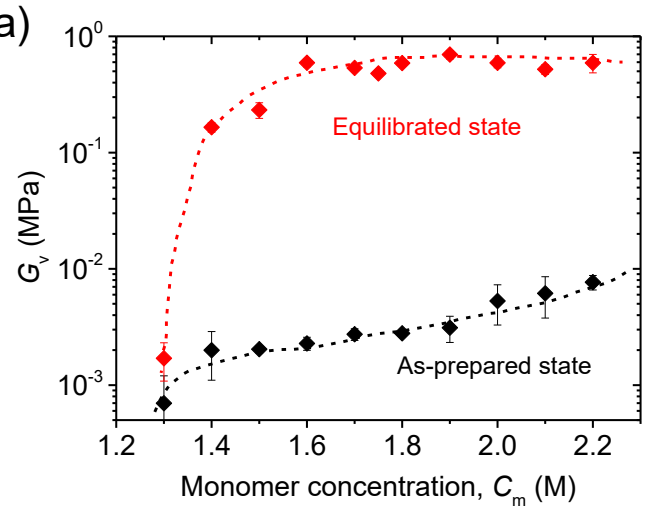

(c)

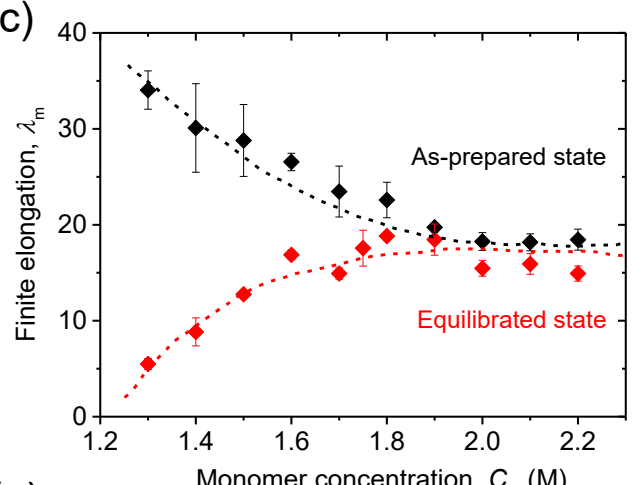

(e)

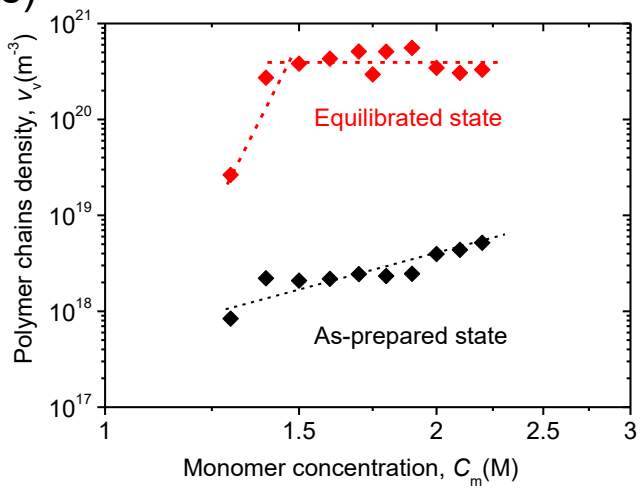

(b)
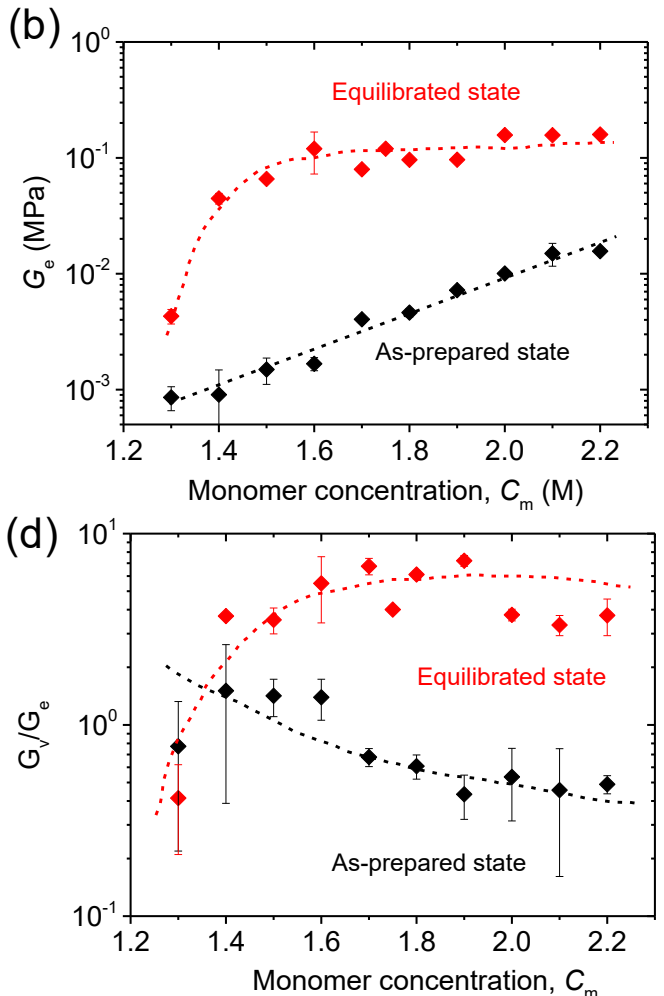

(f)

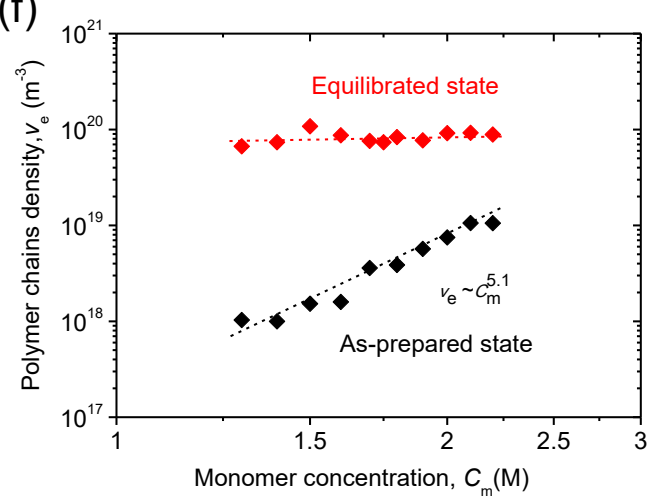

Figure 7. Monomer concentration $C_{\mathrm{m}}$ dependence of shear modulus of viscoelastic part $G_{\mathrm{v}}(\mathrm{a})$, shear modulus of the elastic part $G_{\mathrm{e}}(\mathrm{b})$, theoretical finite fracture elongation $\lambda_{m}$ (c), ratio of $G_{\mathrm{v}} / G_{\mathrm{e}}$ (d) of polyampholyte hydrogels in their as-prepared and equilibrated state. Polymer chain density from the viscoelastic part $v_{\mathrm{v}}(\mathrm{e})$ and elastic part $v_{\mathrm{e}}(\mathrm{f})$. The results were obtained by fitting Figure 2(a, b) with the viscoelastic model. 


\section{Figure 8}
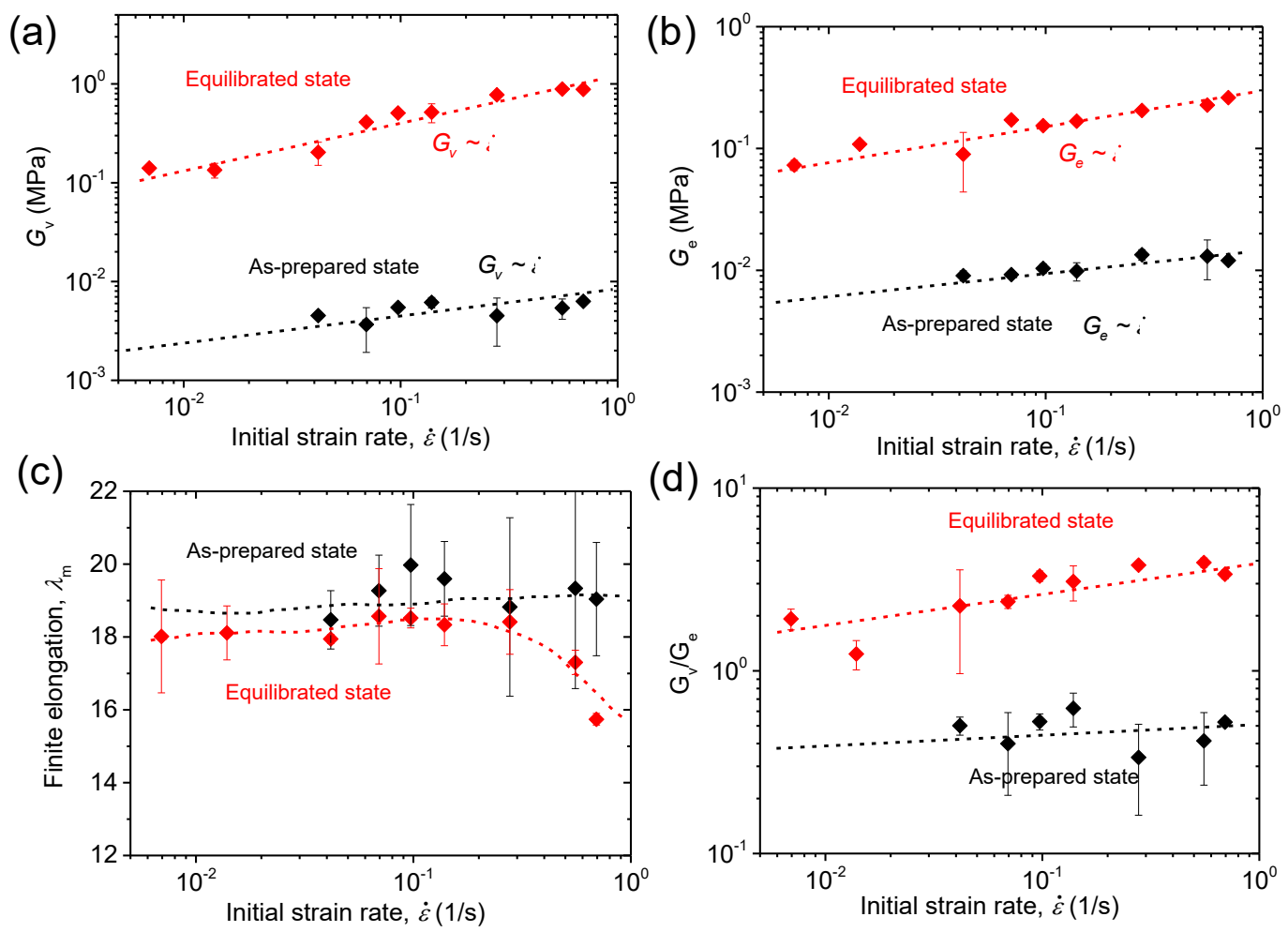

Figure 8 . Initial strain rate $\dot{\varepsilon}$ dependence of shear modulus of the viscous part $G_{\mathrm{v}}$ (a), shear modulus of the elastic part $G_{\mathrm{e}}(\mathrm{b})$, theoretical finite fracture elongation $\lambda_{m}(\mathrm{c})$, ratio of $G_{\mathrm{v}} / G_{\mathrm{e}}(\mathrm{d})$ of a polyampholyte hydrogel $\left(C_{m}=2.1 \mathrm{M}\right)$ in its as-prepared and equilibrated state. The results were obtained by fitting Figure $4(\mathrm{a}, \mathrm{b})$ with the viscoelastic model. 


\section{Figure 9}

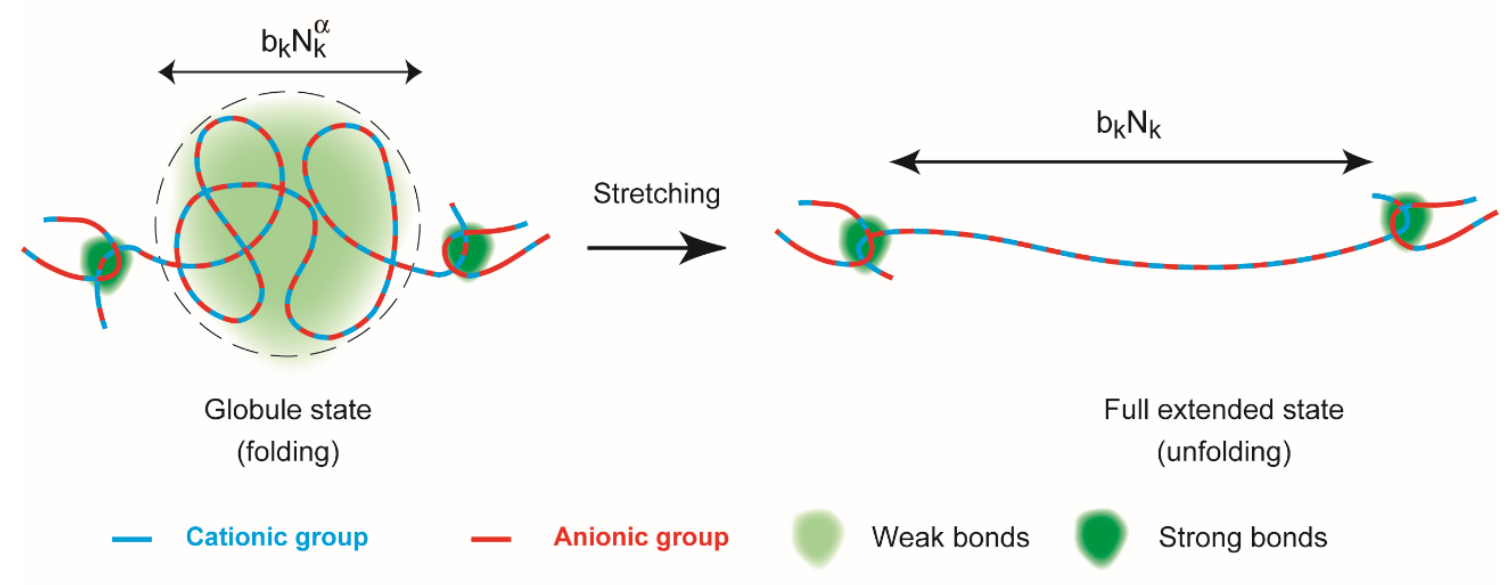

Figure 9 Illustration for the conformation of a partial polyampholyte chain between quasi-permanent crosslinking points. At non-stretching state (left), the polymer chain takes a globule conformation with a size $\mathrm{R}=b_{k} N_{k}^{\alpha} \quad(\alpha=0.4)$. At the ultimate-stretching state (right), the size of the polyampholyte chain becomes $b_{k} N_{k}$. Here $b_{k}$ and $N_{k}$ are the Kuhn length and Kuhn number, respectively. 


\section{Figure 10}

(a)

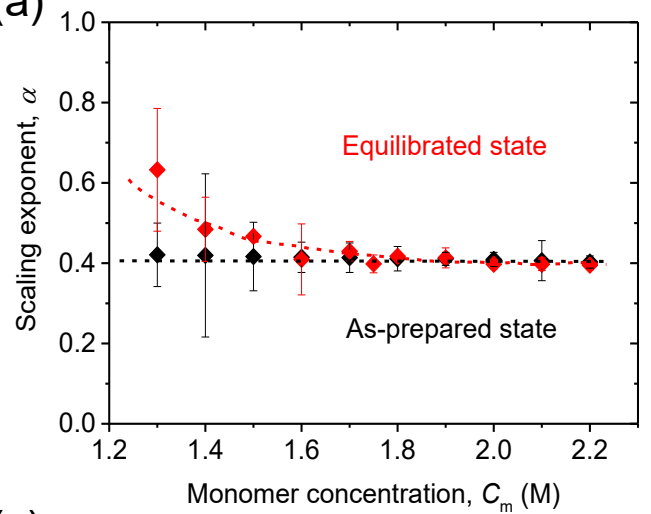

(c)

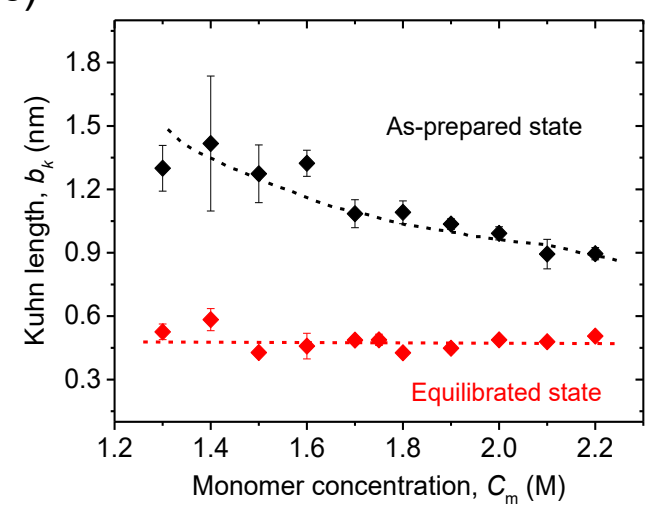

(b)

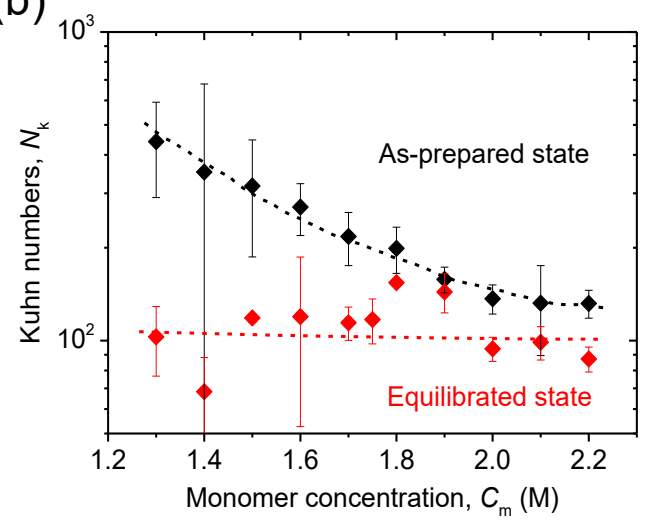

Figure 10. Monomer concentration $C_{\mathrm{m}}$ dependence of the structure parameters of the partial chain between the quasi-permanent crosslinkers of PA gels in the as-prepared and equilibrated state. (a) scaling exponent $\alpha$, (b) Kuhn number $N_{\mathrm{k}}$, (c) Kuhn length $b_{\mathrm{k}}$. 


\section{Figure 11}

(a)

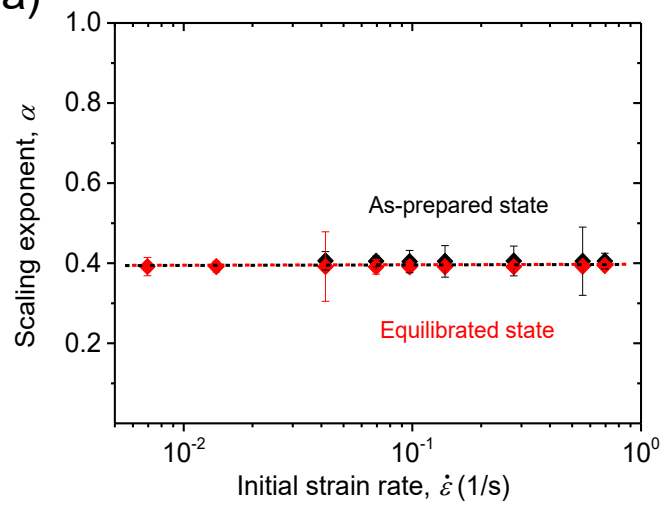

(c)

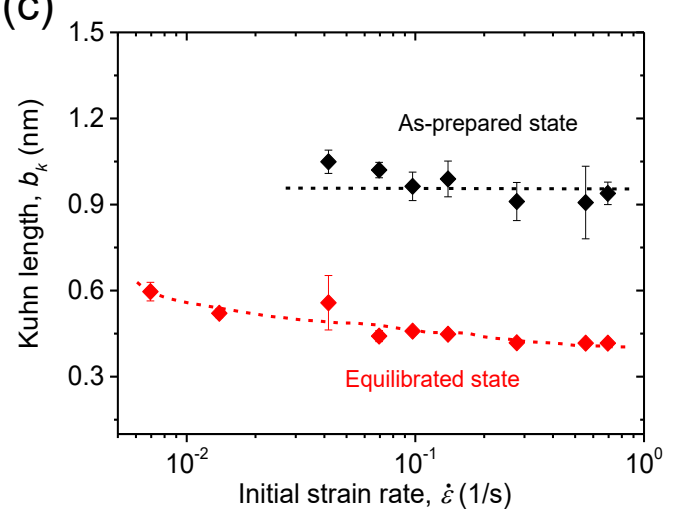

(b)

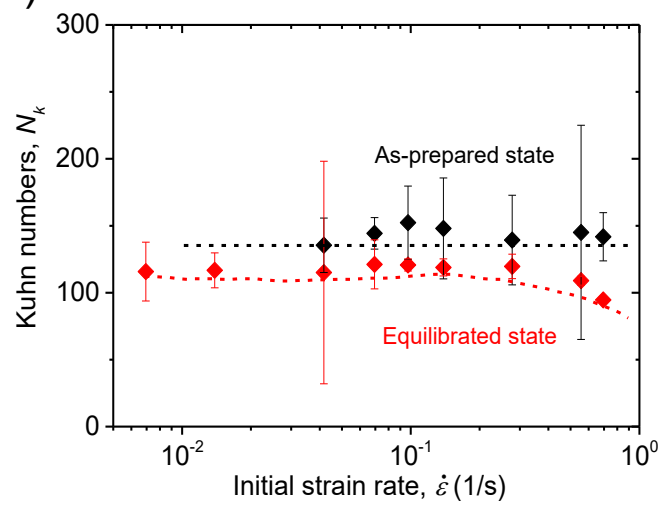

Figure 11. Initial strain rate $\dot{\varepsilon}$ dependence of $\alpha$ (a), $N_{\mathrm{k}}$ (b), and $b_{\mathrm{k}}$ (c) of a polyampholyte hydrogel $\left(C_{m}=2.1 \mathrm{M}\right)$ in its as-prepared and equilibrated state. 


\section{Figure 12}
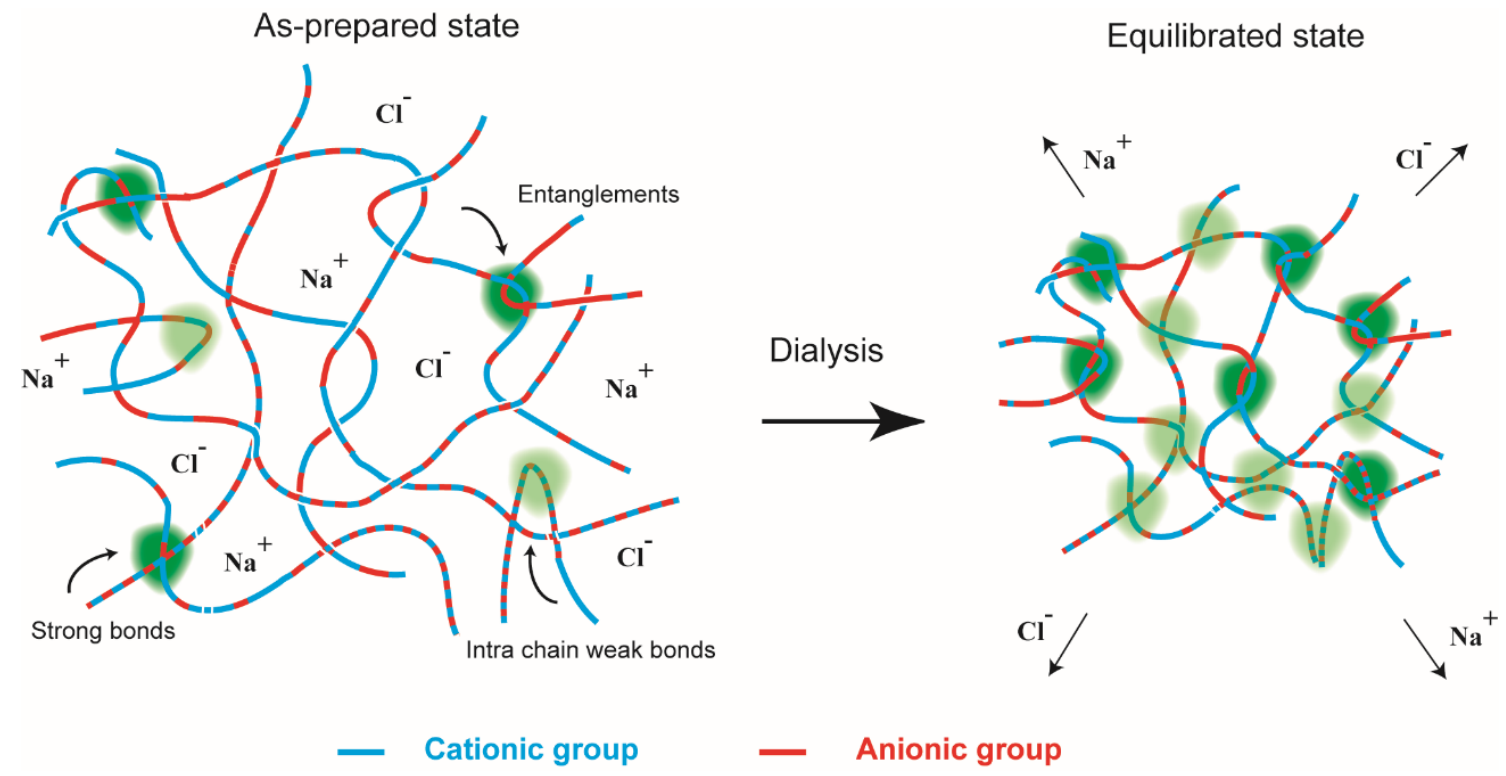

Figure 12. A simplified illustration representing the essential crosslinking feature of polyampholyte gels formed by multiple-ionic-bonds of wide strength distribution in the as-prepared state and equilibrated state. A strong bond refers a multiple-ionic-bonds that does not rupture at the given observation time, while a weak bond refers that ruptures.

The details are described in the conclusions section of text. 